\title{
The problem of racemization in drug discovery and tools to predict it.
}

\author{
Abstract \\ Introduction \\ Racemization has long been an ignored risk in drug development, probably because of \\ a lack of convenient access to good tools for its detection and an absence of methods to \\ predict racemization risk. As a result, the potential effects of racemization have been \\ systematically underestimated.
}

Areas covered

Herein, the potential effects of racemization are discussed through a review of drugs for which activity and side effects for both enantiomers are known. Subsequently, drugs known to racemize are discussed and we review methods to predict racemization risk. Application of a method quantitatively predicting racemization risk to databases of compounds from the medicinal chemistry literature shows that success in clinical trials is negatively correlated with racemization risk.

\section{Expert opinion}

It is envisioned that a quantitative method of predicting racemization risk will remove a blind spot from the drug development pipeline. Removal of the blind spot will make drug development more efficient and result in less late-stage attrition of the drug pipeline.

Keywords

Racemization; quantitative prediction; stability; enantiomers; risk; 


\section{Introduction}

It is generally accepted that enantiomers of chiral compounds can have very different effects in chiral environments. Patients are an intrinsically chiral environment, and in drug development we therefore distinguish the enantiomers of a drug as the eutomer (the good enantiomer) and the distomer (the unwanted enantiomer). It is considered desirable practice to administer chiral compounds as single enantiomers and to profile the properties of both enantiomers before regulatory approval. In fact, even drugs that are initially administered as the racemate (the 50:50 mixture of the two enantiomers) can be considered for a so-called "chiral switch", i.e. development of a singleenantiomer alternative.

Racemization is the process whereby a single enantiomer is converted into a mixture of both enantiomers. Thus, if a compound that tends to racemize is administered as a drug, the patient will over time very probably be exposed to both enantiomers even if the original preparation was a single enantiomer. Racemization is a particular concern to drug discovery because, as mentioned above, the two enantiomers are highly likely to have different biological properties, particularly in vivo. A process related to racemization is that of enantiomerization, which refers to one enantiomer being stereoselectively converted into the other and which can also lead towards a mixture of the two enantiomers.

The impact of chirality on drug discovery has been reviewed previously and here we focus primarily on work that includes specific studies of the process of racemization. 
Relevant reviews include general surveys of chiral drugs, as well as those that describe the impact of chirality on toxicity and on synthesis.[1-5] Some studies of racemization have also been reviewed elsewhere.[6, 7] In the context of dynamic kinetic resolution for the preparation of highly enantio-enriched materials, a rapid racemization is often desirable.[8] Where an enzyme is the chiral catalyst, racemization must occur in conditions that are compatible with the enzyme. These conditions are likely to be similar to those in biological systems in general, and therefore also to be relevant to drug discovery.

In the following sections we first describe studies that illuminate the impact that racemization has on drug safety and efficacy. This is followed by a description of studies that have investigated the process of racemization in biologically relevant conditions. Methods to predict racemization are then described and applied to a set of approved drugs and clinical candidates.

\section{The problem of racemization.}

Although it is known and understood that the two enantiomers of a molecule must experience a different response to any chiral environment (such as a protein), this difference could be large or small. If the difference were small then the divergent biological properties of enantiomers might be safely ignored in all but the most extreme cases and racemization would therefore be of little concern. Understanding this difference between enantiomers is therefore critical to determining the importance of racemization. There are many relevant reports, including some systematic surveys and many case studies, several of which we describe here.[9] 


\subsection{Case studies detailing the differences and similarities between the activity and safety of enantiomers of individual drugs.}

We first review the literature on drugs and drug candidates for which differences in activity for the two enantiomers are known.

$<<$ Figure 1 approximately here >>

Compounds where the safety of the two enantiomers has been investigated are discussed first. One of the most well-known examples of a drug with dangerously different activity between the two enantiomers is thalidomide, 1. The S-enantiomer is believed to be teratogenic.[10, 11] The pharmacokinetics of the two enantiomers also differ, with $S \mathbf{- 1}$ being eliminated faster than $R \mathbf{- 1}$ in humans.[12] However, $S \mathbf{- 1}$ is absorbed more quickly. $S$-Clopidogrel, 2, is an anti-platelet medication that is administered as a pro-drug.[13] It undergoes several key metabolic steps in vivo. While the $R$-enantiomer is inactive, at high doses it can cause convulsions. The harmful effects of the distomers of 27 drugs were summarized in a review from 2007.[14] At the most benign, the distomer is inactive but toxicity is noted for thalidomide (1), penicillamine (3), propranolol (4), vigabatrin (5), tramadol (6) and 2-[2,6-dioxopiperidine-3-yl]phthalimidine (7).[14] Levobupivacaine, R-8, is less cardiotoxic than racemic bupivacaine.[15] This reduced cardiotoxicity prompted a stereoselective synthesis to be developed that involved a resolution and so racemization was desired in order to permit the undesired enantiomer to be recycled into levobupivacaine. High 
temperatures $\left(138^{\circ} \mathrm{C}\right)$ and the addition of ethylene glycol to the aqueous system were found to promote almost complete racemization within 9 hours.

The relative efficacy of the two enantiomers has also been investigated for several compounds. Cetirizine, $\mathbf{9}$, is an antihistamine by virtue of being a selective antagonist of the H1-receptor. It is sold as a racemate which raised the possibility that a chiral switch could see an improved single stereoisomer version (which was subsequently pursued).[16] A decision tree for making this choice was proposed based on the difference in pharmacokinetics and pharmacodynamics of the enantiomers and their comparison to those for the racemate. Levocetirizine was found to have 2-fold higher apparent affinity for the H1-receptor than the racemate;[17] this presumably results from the concentration of levocetirizine accounting for half of the concentration of racemic cetirizine. The affinity of dextrocetirizine was 30-fold lower than for the racemate. The difference between the affinity of the two enantiomers is accounted for by the longer dissociation half-life of the levocetirizine. In trials in healthy volunteers, levocetirizine was as effective as the racemate but dextrocetirizine was less effective. Although the maximum effect for levocetirizine occurred at 6 hours after dosing, at 32 hours the response to levocetirizine was statistically superior to that for the racemate. Low levels of metabolism were observed for both enantiomers but the volume of distribution was higher for the distomer, dextrocetirizine. It was suggested that this higher volume of distribution might make dextrocetirizine more likely to cause unwanted side-effects. The very comprehensive package of data for cetirizine was used to support a case for the use of single enantiomer levocetirizine and reflects the importance of understanding stereochemical detail for a candidate drug. 
Adrenaline, 10, can be used to induce vasoconstriction at the site of injection of local anaesthetics and the L-form is 10 times more effective than the D-form.[18] Certain aromatase inhibitors, such as fadrozole, 11, have significantly different activities for each of the two enantiomers; $S$-fadrozole is most active for instance.[19]

Other studies have investigated the pharmacokinetic properties of enantiomers. Ketoprofen, 12, is a non-steroidal anti-inflammatory and its delivery via a trans-dermal route has been considered.[20] When used orally, it is delivered as a racemate but stereoselective skin permeation might favour its use as a single enantiomer for this alternative delivery route. Studies showed the racemate to be higher melting than the individual enantiomers and $\mathrm{x}$-ray diffraction patterns support the racemate having a different solid form than the individual enantiomers suggesting that the two enantiomers co-crystallize and are both present in the unit cell. When $0.05 \mathrm{M}$ solutions of each of the enantiomers was applied to mouse skin, no difference in permeation was detected between enantiomers or racemate. Further studies supported no stereoselectivity for skin permeability for ketoprofen.

Ketorolac, 13, was investigated in a pharmacokinetic study in healthy volunteers.[21] Following intravenous injection, the concentration of the two enantiomers was monitored separately. This showed that $S$-ketorolac is cleared more rapidly than the $R$ enantiomer leading to higher exposure to $R$-ketorolac.

\subsection{Surveys}


In addition to investigating the differences between properties of individual compounds, larger scale data analyses have also been performed to determine general trends. In one such survey, all of the compounds in AstraZeneca's corporate database where each enantiomer had separately had a key biological property measured were identified.[22] These properties included lipophilicity, solubility, permeability, plasma protein binding, metabolism by microsomes, metabolism by hepatocyte cells, inhibition of certain cytochrome P450 enzymes, block of the hERG cardiac potassium channel and in vivo pharmacokinetic parameters. Most of the properties (apart from $\log \mathrm{D}$ ) required logarithmic transformation to put them onto a linear scale. A statistical analysis of the difference in measured properties between enantiomers was compared to the difference found when measurements were repeated on the same compound. This analysis permitted identification of properties where there is a difference between enantiomers that exceeds the uncertainty in the measurement. In Figure 2A, any property that has a bar adjacent to it is one where enantiomers are found to differ. Inhibition of the cytochrome P450 (CYP) enzymes and in vivo pharmacokinetic parameters (volume of distribution and clearance) are prominent examples. Metabolism, as measured in either hepatocyte cells or microsomal preparations, shows a difference between enantiomers as does binding to plasma proteins and block of the hERG channel. As expected, solubility and $\log \mathrm{D}$ do not show a difference between enantiomers. Surprisingly, in assays to assess permeability through cells or efflux (an active process mediated by membrane-bound proteins) no significant difference between enantiomers was found.

$<<$ Figure 2 approximately here >> 
A similar analysis by researchers at Novartis compared the difference between enantiomers to that between repeat measurements.[23] The distribution of both was compared using the Kolmogorov-Smirnov test and the properties examined were solubility, permeability, CYP inhibition, metabolism in rat microsomes, hERG block and biochemical activity in a randomly selected primary potency screen (isocitrate dehydrogenase, IDH). In addition to testing for whether the distributions are distinct, the authors also assessed whether the difference was likely to be of practical relevance. A p-value assessed whether the distributions are statistically distinct and the authors quantify the difference as the D-value. Figure $2 \mathrm{~B}$ shows the parameters that achieve this difference and the corresponding D-values are shown as solid bars. Parameters affected by chirality include inhibition of IDH, microsomal metabolism and hERG block. They then did a further test to determine whether the difference is likely of practical relevance (assumed to be the case if the D-value, plotted in Figure 2B, was above 0.1). On these grounds they suggest that, although in agreement with the AstraZeneca findings for hERG block on purely statistical grounds, the difference in hERG block by different enantiomers is unlikely to be of practical importance. When a compound blocks the hERG channel, it is possible that this can lead to death of an otherwise healthy patient.[24] We are therefore unsure whether to accept these authors' assessment of relevance. More intriguing is inhibition of CYP3A4. For CYP3A4, the AstraZeneca analysis supports a small but significant difference, whereas the Novartis data suggests a difference that is not significant but which might be large enough to be important. In both cases, CYP3A4 is of borderline significance and so it may not be surprising that the final classifications differ. Both hERG and CYP 3A4 are often characterized as being flexible proteins with large binding cavities and it may be that this leads to weak differentiation of enantiomers.[25, 26] 


\section{3) Measurements of rates of racemization.}

Having confirmed the potential for significantly different effects of enantiomers when used as drugs, both for individual compounds and in general, we now review reports where racemization has been studied.

\subsection{Generalized racemization mechanisms}

In general, there are two dominant mechanisms by which compounds can racemize in aqueous solutions. These mechanisms are deprotonation of a carbon acid under basic conditions and dehydration of an alcohol under acidic conditions (Figure 3A and B).

Other mechanisms do occur but are far less general. An example of an alternative mechanism is provided by the racemization of allantoin which racemizes via both the usual deprotonation and via an intramolecular nucleophilic attack (Figure 3C).[27]

\subsection{Examples of racemizing drugs}

Studies of the racemization of several drugs have been published and we now summarize these reports.

$<<$ Figure 3 approximately here>> 
Initial studies showed that thalidomide 1 racemizes in phosphate buffer (half life of 260 - 290 minutes) and does so even more rapidly in citrated human plasma (half life 8 12 minutes) and that a similar half life is found for defatted human serum albumin (HSA) in phosphate buffer. Presumably racemization of $\mathbf{1}$ proceeds via the generalbase-mediated process shown in Figure 3A. Rat liver microsomes did not promote the racemization.[28] Such studies were extended to show that HSA is mildly stereoselective and promotes the racemization of $\boldsymbol{S} \mathbf{- 1}$ slightly more than it does $\boldsymbol{R}-\mathbf{1}$.[11] The rate of racemization in phosphate buffer was related to the buffer strength. The ability of various amino acids to catalyse racemization was also investigated and showed that the basic amino acids lysine and arginine were more catalytically active than acidic or neutral amino acids. It was noted that albumin incorporates many basic amino acids, including some in the known binding sites and some of these basic groups presumably facilitate the observed racemization. This catalysis is fairly non-specific but is reduced in vivo when many of albumin's binding sites are occupied by molecules such as fatty acids and bilirubin. Since the compound readily racemizes in vivo, it is now known that the tragic consequences of this compound's use during the 1950s could not have been avoided by providing patients with a single enantiomer.[10, 11]

Studies of the racemization of $\mathbf{2}$ in vitro needed to include hepatocytes, because metabolism and the formation of conjugates are likely to be important in any racemization in vivo.[13] In vivo studies were also performed and suggested that the acid is the predominant circulating metabolite and that $4-8 \%$ of the acid is the $R$ enantiomer. In vitro, the half life of racemization was found to be in the range 3.5 to 8.5 weeks with a dependency on the buffer and the methanol concentration (methanol was required to improve solubility). Complementary studies investigated isotope 
exchange in $\mathrm{D}_{2} \mathrm{O}$. Even with the hepatocytes present, less than $2 \%$ inversion was detected and so no satisfactory explanation was provided for the level of racemization measured in vivo. We have subsequently also studied racemization of $\mathbf{2}$ and found that racemization of $\mathbf{2}$ in vitro is likely to appear much slower than it is in reality because of the very poor solubility of $\mathbf{2}$ limiting the amount of free $\mathbf{2}$ in solution.

The discussion of the racemization of 27 drugs from 2007 mentioned above reviewed several studies of racemization, including the possibility of racemization being mediated by degradation and bioconjugation (such as the formation of S-CoA thioesters).[14]

The requirement for extreme conditions for racemization of levobucaine (R-8) in its dynamic kinetic resolution (vide supra) make racemization unlikely to occur in vivo.

In trials in healthy volunteers, using radiolabelled levocetirizine R-9, it was found that the total concentration of cetirizine was equivalent to the concentration of levocetirizine and no dextrocetirizine was found in urine. Both of these observations support the conclusion that there is no in vivo racemization, in agreement with observations in $\mathrm{pH}$ 7.4 buffer.[16]

Considering the significant difference in activity between L- and R-adrenaline, racemization would significantly reduce activity. Studies of a range of products including those mixed with anaesthetics found racemization of adrenaline (10) to be slow.[18] 
To investigate the racemization of fadrozole (11), a set of $\mathrm{N}$-substituted imidazoles were investigated.[19] It was found that two of the compounds racemize measurably in aqueous buffer at $\mathrm{pH} 7.4$ and $\mathrm{pH} 9.6$ with longer half lives at $\mathrm{pH} 7.4$ supporting a general-base-catalysed process (Figure 3A). Studies in bovine serum albumin suggested that this medium had little effect.

In the studies where ketoprofen (12) was applied to mouse skin, no racemization was detected.[20]

Ketorolac (13) is a non-steroidal anti-inflammatory agent sold as a racemic mixture. Its racemization is found to be $\mathrm{pH}$ dependent and to be slowest at intermediate $\mathrm{pHs}$ (3.0 - 7.5).[29, 30] A mechanism for racemization involving deprotonation of the already anionic carboxylate at high $\mathrm{pH}$ and protonation of the neutral acid at low $\mathrm{pH}$ is proposed (Figure 3D).

Litronesib, 14a, is an investigational compound that inhibits $\mathrm{Eg} 5$ and has been investigated as a treatment for several types of malignancy.[31] An unexpected loss of chiral integrity was observed upon storage of this compound. The chiral centre in this case is fully substituted and so the racemization was surprising. Studies in tartrate solutions across a $\mathrm{pH}$ range from 2.1 to 8.2 and using other buffers suggested that $\mathrm{pH}$ is the key factor rather than buffer identity. Extensive studies led to the proposal of the ylid-mediated path shown in Figure 3E. The analogues $14 \mathrm{~b}$ and $14 \mathrm{c}$ helped elucidate the mechanism; $\mathbf{1 4 b}$ is racemized about twice as fast and $\mathbf{1 4 c}$ three times as slowly as 14a, consistent with the ylid structure shown. 
Agonists of the $\beta 2$ adrenoceptor are often chiral benzyl alcohols with activity usually resting predominantly with the $R$-enantiomer.[32] An example of such a molecule is meluadrine, 15. Studies of $\mathbf{1 5}$ led to the conclusion that across the $\mathrm{pH}$ range the mechanism of racemization involves elimination of $\mathrm{OH}$ followed by rehydration. Meanwhile, the details concerning the protonation of the base and alcohol vary (Figure 3F). Arrhenius plots at various temperatures suggested an activation energy in the range $95-115 \mathrm{~kJ} / \mathrm{mol}$. Other studies of the stability of chiral benzyl alcohols have described in detail the process by which the leaving alcohol must leave the solvent cage and join bulk water to permit access to the planar carbocation intermediate that would be required for racemization.[33]

A purely computational study investigated the possibility of inversion at sulfur in the proton pump inhibitor esomeprazole (16).[34] A mechanism in which the pyramidal sulfur becomes planar was studied and the barrier to inversion computed to be prohibitively high $(180 \mathrm{~kJ} / \mathrm{mol})$ suggesting that very high temperatures would be required for this to operate.

An innovative method using three different regions in a flow set up permitted separation of enantiomers, racemization and analysis within a closed system.[35] This method was used to study $\mathbf{1 7}$. The mechanism of racemization was proposed to involve a ring opening to an intermediate that also provided access to a hydrolysis product (Figure 3G). We propose (given $\mathrm{p} K_{\mathrm{a}}$ considerations) an alternative intermediate, which proceeds via a cleavage in which the sulfonamide acts as leaving group. 
The racemization of mandelic acid (18) was found to proceed faster than the rate of deuterium incorporation under acidic conditions.[36] It is proposed that under these conditions, racemization (or possibly enantiomerization) occurs via protonation of the hydroxyl with displacement by water. By contrast, in neutral and basic conditions, the rates are the same and the racemization proceeds via the general-base-catalysed process involving the enol(ate) (Figure 3H).

Amfepramone (19) is a stimulant that can be used as an appetite suppressant and is usually used as a racemate.[37] The rate of racemization was studied as buffer strength, ionic strength, temperature and drug concentration were varied. The dependence on $\mathrm{pH}$ and on buffer strength revealed non-linear relationships. Cyclodextrin additives were also investigated for their ability to suppress racemization but showed mixed results with most examples actually promoting racemization while only a small number were protective. In human plasma, the half life for racemization was measured to be $23-25$ minutes but this is much lower than the known in vivo half life following oral dosing of 1.5 to 2 hours. Studies in phosphate buffers at a range of pHs and buffer strengths support a general-base-catalyzed reaction (Figure 3A) for 19 and the analogous primary amine.

As part of a search for safe versions of thalidomide, the rate of enantiomerization of 7 and of lenalidomide $\mathbf{2 0}$ were measured alongside those for $\mathbf{1}$ (thalidomide) in $50 \mathrm{mM}$ phosphate buffer at $\mathrm{pH}$ 8.[38] Although it is likely that these compounds racemize, like thalidomide, the use of dynamic electrokinetic chromatography means that only the enantiomerization can be observed. By investigating the variation with temperature, the activation parameters were derived and revealed that at $298 \mathrm{~K}$ the Gibbs free energy 
barrier $\left(\Delta G^{\dagger \ominus}\right.$ ) for enantiomerization of $\mathbf{1}$ is $101.7 \mathrm{~kJ} / \mathrm{mol}$ (with $\Delta H^{\dagger \ominus} 99.0 \mathrm{~kJ} / \mathrm{mol}$ ), for 7 the barrier is $96.8 \mathrm{~kJ} / \mathrm{mol}$ (with $\Delta H^{\dagger \ominus} 46.1 \mathrm{~kJ} / \mathrm{mol}$ ) and for 20 the barrier is $91.5 \mathrm{~kJ} / \mathrm{mol}$ (with $\Delta H^{* \ominus} 62.4 \mathrm{~kJ} / \mathrm{mol}$ ). This suggests that these other examples are more likely to racemize than thalidomide itself.

The half life for racemization of $\mathbf{2 1}$ was studied in a range of media.[39] A surprising dependence on $\mathrm{pH}$ was observed in which the rate was fastest at $\mathrm{pH}$ 6, slowest at $\mathrm{pH} 9$ and intermediate at pHs 4 and 7. In all cases, the half life was less than 2 hours. Rapid racemization (half life of a few minutes) was also measured in dog plasma. Although no mechanistic proposals are made, it seems realistic to imagine that this compound could racemize through an acid-catalysed ring-opening ring-closing mechanism or through a base-catalysed process (as for Figure 3A) and this would reasonably lead to a complicated dependence on $\mathrm{pH}$.

\subsection{Racemization of model drugs}

A significant expansion of the dataset of aqueous racemization rates has been described in recent publications.[6, 40-42] The rates of racemization of 28 different model compounds racemizing by the base-catalysed pathway were reported. These included a series of compounds with chiral centres bearing an $\mathrm{N}$-linked amide and an aromatic ring with either a C-linked amide or a C-linked ester as the third substituent (22 and 23). A further set included hydantoins (24) and thiohydantoins (25) (some hydantions had been studied previously).[43] The rate of racemization in phosphate buffer was studied and the second-order rate constant for the general-base-catalysed racemization 
of each compound was obtained. These were measured using a range of experimental techniques, including circular dichroism spectroscopy and monitoring deuteration in $\mathrm{D}_{2} \mathrm{O}$ using ${ }^{1} \mathrm{H}$ NMR spectroscopy. The second-order rate constants were found to span over 4 orders of magnitude and to bracket rates likely to be of biological relevance.

\section{Tools to predict racemization.}

In a thorough review of stereochemistry and racemization, Testa provided a table that defines the types of group that can either increase or decrease configurational stability (Table 1).[9, 44] He suggested that stereochemical instability requires the presence of at least one of the groups that decrease stability and the absence of any of the groups that increase stability. This provides a rule of thumb that can easily be kept in mind by chemists designing new molecules but has a narrow scope and is acknowledged to be based on limited evidence for certain groups.

$<<$ Table 1 approximately here>>

This approach was updated in 2018 with two computational approaches paired with two experimental approaches.[6] In all cases, any stereogenic carbon that has a proton attached to it can be analysed with an assumption that the most likely mechanism of racemization for such compounds will be general-base-catalysed deprotonation to give an anionic intermediate (Figure 3A). The computational methods both rely on predicting the stability of this anion and correlating this with measured rate constants (both those reported in the same paper and those in the literature that were measured under suitable conditions). The first and most readily applicable of the computational 
methods is based on group contributions. These are shown in Table 2. The contribution of all of the groups is added together. There is a correction if two or more of the groups stabilize the anion via delocalisation; this cross-conjugation correction is +15.0 $\mathrm{kcal} / \mathrm{mol}$. The resulting value $(\sigma)$ can be used to predict the second-order rate constant for racemization via general-base-mediated reaction $\left(k_{\mathrm{gb}}\right)$ using the equation:

$\log \left(k_{\mathrm{gb}}\right)=-0.11 \sigma-9.81$

There is an interesting subset of cases (including compounds like 21, 24 and 25) where the stereogenic centre is in a ring and the reaction can proceed through an anion that is formally aromatic. In such cases, a modified equation is applied:

$\log \left(k_{\mathrm{gb}}\right)=-0.26 \sigma-16.95$

An alternative view is to determine a cut-off value of $\sigma$ where the corresponding rate constant indicates that racemization is likely to be of biological relevance. Comparing values measured in different media suggests that blood might be similar in terms of general-base capacity to phosphate buffer with a concentration of 0.15 M.[12] This leads to the suggestion that if an amount of racemization in a given time period that is thought acceptable can be determined then a threshold for acceptability can be defined. For instance, if $10 \%$ racemization within 24 hours were deemed the acceptable limit then any compound with a sum of contributions below $-44 \mathrm{kcal} / \mathrm{mol}$ should be considered at risk.

$<<$ Table 2 approximately here>> 
The group contribution approach has the power of being applicable very rapidly via implementation as a computational algorithm. It is limited whenever there is cross-talk between the substituents (as in the cross-conjugation correction or the aromatic anion intermediates). In such cases, a quantum mechanical approach can help. It was shown that if the deprotonation energy is computed at the B3LYP/6-31+G** level (including solvation via the PCM protocol) that these energies also correlate with $\log \left(k_{\mathrm{gb}}\right)$.[45-48] The deprotonation energies are computed relative to methane to give a value styled $\Delta \Delta \mathrm{G}$. With this treatment $k_{\mathrm{gb}}$ is obtained using:

$\log \left(k_{\mathrm{gb}}\right)=-0.20 \Delta \Delta \mathrm{G}-14.28$

This quantum mechanical method tends to give values in better agreement with experiment than the more general group-contribution-based method. Applying the same approach to defining a threshold as described above yields a value of -45 $\mathrm{kcal} / \mathrm{mol}$; any compound with a value of $\Delta \Delta \mathrm{G}$ below this should be considered at risk of racemizing.

Finally, experimental studies might be required to confirm a prediction or when the computational methods do not provide consistent predictions. Following racemization by deuterium in deuterated buffers can use ${ }^{1} \mathrm{H}$ NMR or mass spectrometry. Care must be taken to correct for any other side-reactions (typically hydrolyses) and to investigate the influence of buffer concentration. Alternatively, the reaction can be followed directly by circular dichroism. 


\section{Application of the predictive tool to drugs and clinical candidates.}

The European Bioinformatics Institute hosts the ChEMBL database and one of the subsets that they curate is described as "Drugs" including "Approved Drugs" up to March 2018.[49] In addition, the set includes compounds that are under clinical investigation. Compounds are annotated according to the most advanced clinical phase they have reached to date. These are in the range 0 to 4 , where 0 is pre-clinical and 4 is approved. Those compounds that have received at least one approval are annotated with the year of this first approval. They also annotate those compounds that are approved and subsequently withdrawn. We have applied the group contribution method described above to assess the racemization liabilities that are present in this set of compounds.

\subsection{An overview of racemization risk in drugs and drug candidates}

We have identified all those compounds that have a single stereogenic carbon atom in them and which are computed to racemize to at least $10 \%$ within 24 hours. In the first place, the percentage of all of the compounds in each of the clinical phases that these represent has been computed. This is shown in Figure 4A and shows a very clear trend; there are proportionately fewer racemizing compounds as the clinical stages progress. This suggests that compounds that racemize are less likely to succeed in each clinical phase than compounds that do not racemize. This is an interesting countervailing trend to one published previously which shows compounds that include at least one stereocenter are more likely to succeed in the clinic.[50] Racemizing compounds 
therefore appear to be a particular liability in the clinic by decreasing the chance of success despite necessarily including a chiral center.

$<<$ Figure 4 approximately here >>

It is worth noting that among compounds that have made it as far as approval, we find that $1.4 \%$ are likely to racemize which is a higher proportion than is found in all of the clinical phases. The approved compounds have therefore been examined in more detail. The approved compounds in this set are made up of many different types of molecules (Figure 4B), amongst which the largest two groups are synthetic small molecules (1643 compounds) and natural product derived molecules (547 compounds). These are also the only two groups that include compounds that are computed to racemize (although it should be noted that stereochemical scrambling of one center in compounds with several stereogenic centers is predicted to occur in some of the other categories of compound). There is an interesting divergence between the nature of the natural products and the synthetic small molecules in terms of their stereochemical makeup. Natural product derived drugs (Figure 4B bottom right) are overwhelmingly likely to be single stereoisomers (470) as compared to being achiral (27) or racemates (40). By contrast, for synthetic small molecules (Figure 4B bottom left), the largest group of compounds are achiral (820), followed by racemates (388) and then single stereoisomers (339).

In the whole set of approved compounds, we predict that 37 are likely to racemize. Of these only one (Valacyclovir) is from the natural product derived drugs, the remaining 36 are synthetic small molecules. It is unsurprising that 28 of these 37 compounds are 
provided as racemic mixtures; this observation confirms that for these compounds racemization in vivo is sufficiently fast to make the use of a single enantiomer pointless.

Among all of the drugs to have achieved approval, 149 (5.5\%) have subsequently been annotated as withdrawn. Although the set of compounds is too small for the difference to be assessed as significant, 3 of the $37(8.1 \%)$ compounds that are predicted to racemize are annotated as withdrawn .

The 9 compounds that are single stereoisomers but which are predicted to racemize are examined in more detail in the following paragraphs.

\subsection{Compounds predicted at risk of racemization distributed as a single enantiomer}

The compounds sold as single isomers but predicted to racemize are cycloserine (26), bentiromide (27), valacyclovir (28), ropivacaine (29), clopidogrel (2), brinzolamide (30), levobupivacaine (8), levocetirizine (9) and safinamide (31). Two of these nine compounds (27 and $\mathbf{8}$ ) are annotated as discontinued (as distinct from withdrawn). Two (2 and 28) are prodrugs for which the active drug is either achiral (28) or much less likely to racemize ( 2 , as discussed above).

The remaining five compounds are surveyed in more detail. Compound $\mathbf{2 6}$ is an inhibitor of alanine racemase, an enzyme that provides the D-Alanine required for cell wall biosynthesis.[51] It is likely that its ability to racemize is not a problem in this context. Compound $\mathbf{2 9}$ is a local anaesthetic that can be administered intravenously and 
the single isomer version was promoted as providing reduced toxicity and improved efficacy.[52] In this case, studies of $\mathbf{2 9}$ in a range of species suggest that there is no racemization in vivo.[53] Compound $\mathbf{3 0}$ inhibits carbonic anhydrase and is used intraocularly via topical application and hence is likely to be protected from the racemizing medium of the plasma.[54] Antihistamine 9 had its in vivo racemization studied as described above. It is likely that the steric bulk of the three rings (two phenyl and one piperazine) that must become co-planar inhibits racemization in this case. Whereas such effects can be reproduced reasonably well by quantum mechanical calculations, the group-contribution approach that we have used here does not account for the effects of steric hindrance. Finally, $\mathbf{3 1}$ is a relatively recently approved treatment for Parkinson's disease that is used in tablet form for oral administration and for which the two stereoisomers show different profiles but rates of racemization have not been reported.[55]

There is a temporal component to the drug approvals but not all of the compounds have had their approval data added to the database. Of the compounds predicted to racemize, the largest number was approved in the 1990s (Figure 4C). It was in 1992 that the US Food and Drug Administration (FDA) changed their guidance with regards to stereoisomers and required assessment of racemization rate and of the properties of the individual enantiomers. [56] There is no clear trend for changes either in the number of compounds that are likely to racemize being approved or in whether they are approved as single stereoisomers or racemates. The earlier period featured fewer single stereoisomers that are likely to racemize being approved, probably because of a reliance on configurationally stable natural product derivatives. Following the change in FDA 
guidance in 1992, the number and proportion of compounds computed to be likely to racemize that were approved has decreased.

\section{Conclusions}

This review explores the merit of fast quantitative predictions of racemization risk in drug development. We show that racemization risk negatively affects a drug candidate's chance to pass through clinical trials and that ignoring racemization risk therefore introduces an unnecessary risk in the drug development pipeline.

\section{Expert opinion.}

The two enantiomers of a molecule can have quite different biological properties. In particular, the primary potency, pharmacokinetic parameters and certain types of toxicity are likely to be different. This ensures that understanding the properties of the two enantiomers will be important in drug discovery and that the process of racemization will often be undesirable.

\subsection{Comparison with the status quo}

Racemization of drug-like compounds usually proceeds via a mechanism that can be easily understood by chemists. The most common such mechanism is that of generalbase-catalysis via an intermediate, if transient, anion. Ideas of delocalisation, electronwithdrawing groups and steric effects permit the ready post-rationalisation of the occurrence of racemization (either in vivo or during the synthesis of chiral molecules). Unfortunately, the ease with which the past is understood has not yet been translated 
into a ready ability to predict which compounds are likely to racemize in vivo. The status quo thus represents a considerable weakness in drug development.

New computational and experimental tools transform this situation. We advocate the use of three different methods in a tiered approach. A simple group contribution method permits the prediction of rates of racemization with minimal computational cost. For molecules of particular interest, or where there is uncertainty about the prediction, a quantum mechanical calculation can be performed. Both of these methods rely on a close correlation between the stabilisation of the anionic intermediate and the rate of general-base-catalysed racemization. Finally, experimental approaches can be used where isotope exchange is followed by mass spectrometry or racemization by circular dichroism spectroscopy. This three-tiered quantitative approach is considerably more robust than the alternative descriptive approaches. Moreover, the fact that the group-contribution approach is conveniently automated through the use of SMILES descriptors makes it applicable to large databases of compounds, allowing efficient identification of racemization risk.

\subsection{Racemization risk in the current drugs and in the pipeline}

Application of the group contribution method to a database of marketed drugs reveals that compounds that racemize are less likely than other compounds to proceed from one stage of clinical testing to another, and this is despite the fact that compounds that contain a stereogenic center are generally more likely to succeed. Very few compounds that are predicted to racemize have been approved and those that have are predominantly prescribed as racemic mixtures. Our analysis thus suggests that compounds at risk of racemization fare worse than typical chiral compounds. 
Quantitative prediction of racemization risk will allow early identification of compounds at risk, providing an opportunity to avoid significant costs associated with compounds that fail in clinical trials. Quantitative prediction of racemization risk therefore facilitates drug discovery and reduces attrition.

\subsection{Single enantiomer drugs at risk of racemization}

Amongst the few compounds that have been approved as single stereoisomers, but which these calculations predict as likely to racemize, some are prodrugs that render the racemization less relevant. Others have been tested for racemization in vivo with none detected, suggesting that any racemization occurs on a timescale that is longer than the compounds are cleared from systemic circulation. Developing a deeper understanding of which properties have allowed these exceptional compounds to make it to the market is of obvious interest.

\subsection{Quantitative predictions reduce the risks introduced by fallible humans}

It seems clear that drug discovery scientists have been bringing forward compounds for clinical testing which have a tendency to racemize without correctly appreciating the risk of failure and the extra challenges that such molecules present. This behaviour appears to be a typical example of underestimating poorly appreciated risk and neglecting under-appreciated opportunities, which also occurs, e.g., in the case of selfdisproportionation of enantiomers.[57-61] The tools that have recently been described should help pre-empt and avoid problems in the future and it is our strongly held opinion that their use would be a sensible step to take when prioritising a set of compounds for synthesis in a drug discovery project. We envisage that tools quantitatively predicting racemization risk will find a permanent place in the medicinal 
chemist's toolbox alongside other predicted properties, in particular those stemming from the use of artificial intelligence. Such quantitative tools will allow the early identification of risk, supporting a lean and safe drug development pipeline.

\section{Article Highlights}

- Racemization can seriously affect the efficacy and safety of drugs

- Racemization risk can be predicted quantitatively and automatically using a group-additivity based approach.

- Success in clinical trials is negatively correlated with increasing racemization risk.

- Evaluation of racemization risk early on in drug development will reduce latestage attrition of the drug development pipeline. 
1. Smith SW. Chiral toxicology: it's the same thing...only different. Toxicol Sci 2009;110:4-30.

**2. Testa B, Vistoli G, Pedretti A, Caldwell J. Organic stereochemistry. Part 5. Helv Chim Acta 2013;96:747-98.

This review presents an in-depth and complete overview of the general principles of stereochemistry.

3. Ariens EJ. Stereoselectivity of bioactive agents: general aspects. J Med Chem; 1983 1983; 1983. p. 11-32.

*4. Caner H, Groner E, Levy L, Agranat I. Trends in the development of chiral drugs. Drug Discov Today 2004;9:105-10.

This paper discusses trends in drug development with a focus on the effects of the 1992 FDA statement on the development of new stereoisomeric drugs.

**5. Islam MR, Mahdi JG, Bowen ID. Pharmacological importance of stereochemical resolution of enantiomeric drugs. Drug Saf 1997;17:149-65.

This review presents an extensive overview of the possible effects of chirality on pharmacological properties.

6. Ballard A, Ahmad HO, Narduolo S, Rosa L, Chand N, Cosgrove DA, et al. Quantitative prediction of rate constants for aqueous racemization to avoid pointless stereoselective syntheses. Angew Chem Int Ed 2018;57:982-85.

7. Ebbers EJ, Ariaans GJA, Houbiers JPM, Bruggink A, Zwanenburg B. Controlled racemization of optically active organic compounds: Prospects for asymmetric transformation. Tetrahedron 1997;53:9417-76.

8. El Gihani MT, Williams JMJ. Dynamic kinetic resolution. CurrOpinChemBiol 1999;3:11-15. 
**9. Testa B. Organic stereochemistry. Part 2. Helv Chim Acta 2013;96:159-88.

This review presents an in-depth and complete overview of the general principles of stereochemistry with a focus on medicinal sciences.

10. McBride WG. Thalidomide and congenital abnormalities. Lancet 1961;278:1358.

11. Reist M, Carrupt PA, Francotte E, Testa B. Chiral inversion and hydrolysis of thalidomide: mechanisms and catalysis by bases and serum albumin, and chiral stability of teratogenic metabolites. ChemResToxicol 1998;11:1521-28.

*12. Eriksson T, Bjorkman S, Roth B, Fyge A, Hoglund P. Stereospecific determination, chiral inversion in vitro and pharmacokinetics in humans of the enantiomers of thalidomide. Chirality 1995;7:44-52.

This paper provides rare, and therefore very valuable, kinetic data for racemization in humans.

13. Reist M, Roy-de Vos M, Montseny JP, Mayer JM, Carrupt PA, Berger Y, et al. Very slow chiral inversion of clopidogrel in rats: a pharmacokinetic and mechanistic investigation. Drug MetabDispos 2000;28:1405-10.

**14. Ali I, Gupta VK, Aboul-Enein HY, Singh P, Sharma B. Role of racemization in optically active drugs development. Chirality 2007;19:453-63.

The authors present a detailed overview of studies of racemization of optically active drugs.

15. Langston M, Dyer UC, Frampton GAC, Hutton G, Lock CJ, Skead BM, et al. Racemisation of R-bupivacaine: A key factor in the integrated and economic process for the production of levobupivacaine. Org Process Res Dev 2000;4:530-33. 
16. Tillement JP, Testa B, Bree F. Compared pharmacological characteristics in humans of racemic cetirizine and levocetirizine, two histamine $\mathrm{H}(1)$-receptor antagonists. Biochem Pharmacol 2003;66:1123-26.

17. Gillard M, Perren CVD, Moguilevsky N, Massingham R, Chatelain P. Binding characteristics of cetirizine and levocetirizine to human $\mathrm{H} 1$ histamine receptors: contribution of Lys191and Thr194. Mol Pharmacol 2002;61:391-99.

18. Sanger-van de Griend CE, Ek AG, Widahl-Nasman ME, Andersson EKM. Method development for the enantiomeric purity determination of low concentrations of adrenaline in local anaesthetic solutions by capillary electrophoresis. J Pharm Biomed Anal 2006;41:77-83.

19. Danel C, Foulon C, Goossens J-F, Bonte J-P, Vaccher C. Kinetics of racemization of enantiopure $\mathrm{N}$-imidazole derivatives, aromatase inhibitors: studies in organic, aqueous, and biomimetic media. Tetrahedron: Asymmetry 2006;17:2317-21. 20. Kommuru TR, Khan MA, Reddy IK. Racemate and enantiomers of ketoprofen: Phase diagram, thermodynamic studies, skin permeability, and use of chiral permeation enhancers. J Pharm Sci 1998;87:833-40.

21. Campanero MA, Lopez-Ocariz A, Garcia-Quetglas E, Sadaba B, Azanza JR. Determination of ketorolac enantiomers in plasma using enantioselective liquid chromatography. Application to pharmacokinetic studies. Chromatographia 1998;48:203-08.

22. Leach AG, Pilling EA, Rabow AA, Tomasi S, Asaad N, Buurma NJ, et al. Enantiomeric pairs reveal that key medicinal chemistry parameters vary more than simple physical property based models can explain. MedChemCommun 2012;3:52840. 
23. Bagdanoff JT, Xu Y, Dollinger G, Martin E. Effect of chirality on common in vitro experiments: an enantiomeric pair analysis. JMedChem 2015;58:5781-88.

24. Sanguinetti MC, Tristani-Firouzi M. hERG potassium channels and cardiac arrhythmia. Nature (London, U K) 2006;440:463-69.

25. Zachariae U, Giordanetto F, Leach AG. Side chain flexibilities in the human ether-a-go-go related gene potassium channel (hERG) together with matched-pair binding studies suggest a new binding mode for channel blockers. J Med Chem 2009;52:4266-76.

26. Ekroos M, Sjoegren T. Structural basis for ligand promiscuity in cytochrome $\mathrm{P}$ 450 3A4. Proc Natl Acad Sci U S A 2006;103:13682-87.

27. Kahn K, Tipton PA. Kinetics and mechanism of allantoin racemization. Bioorganic Chemistry 2000;28:62-72.

28. Knoche B, Blaschke G. Investigations on the in-vitro racemization of thalidomide by high-performance liquid-chromatography. J Chromatogr A 1994;666:235-40.

29. Brandl M, Conley D, Johnson D, Johnson D. Racemization of ketorolac in aqueous solution. J Pharm Sci 1995;84:1045-48.

30. Reist M, Christiansen LH, Christoffersen P, Carrupt P-A, Testa B. Low configurational stability of amfepramone and cathinone: Mechanism and kinetics of chiral inversion. Chirality 1995;7:469-73.

31. Baertschi SW, Jansen PJ, Montgomery RM, Smith WK, Draper JR, Myers DP, et al. Investigation of the mechanism of racemization of litronesib in aqueous solution: unexpected base-catalyzed inversion of a fully substituted carbon chiral center. J Pharm Sci 2014;103:2797-808. 
32. Matsuo K, Yamamoto Y, Kado N, Yamazaki M, Nagata O, Kato H, et al. Racemization kinetics of meluadrine tartrate in aqueous solution. Chem Pharm Bull 2001;49:101-04.

33. Filippi A, Gasparrini F, Speranza M. Hindered inversion of chiral ion-dipole pairs. J Am Chem Soc 2001;123:2251-54.

34. Bruni AT, Castro Ferreira MM. Theoretical study of omeprazole behavior: Racemization barrier and decomposition reaction. Int $\mathrm{J}$ Quantum Chem 2008;108:1097-106.

35. Cannazza G, Battisti U, Carrozzo MM, Brasili L, Braghiroli D, Parenti C. Evaluation of stereo and chemical stability of chiral compounds. Chirality 2011;23:851-59.

36. Chiang Y, Kresge AJ, Popik VV, Schepp NP. The mandelic acid keto-enol system in aqueous solution, generation of the enol by hydration of phenylhydroxyketene and phenylcarboxycarbene. J Am Chem Soc 1997;119:1020312.

37. Mey B, Paulus H, Lamparter E, Blaschke G. Kinetics of racemization of (+) and (-) - diethylpropion: Studies in aqueous solution, with and without the addition of cyclodextrins, in organic solvents and in human plasma. Chirality 1998;10:307-15.

38. Walz S, Weis S, Franz M, Rominger F, Trapp O. Investigation of the enantiomerization barriers of the phthalimidone derivatives EM12 and lenalidomide by dynamic electrokinetic chromatography. Electrophoresis 2015;36:796-804.

39. Welch CJ, Kress MH, Beconi M, Mathre DJ. Studies on the racemization of a stereolabile 5-aryl-thiazolidinedione. Chirality 2003;15:143-47.

40. Ballard A. Kinetics and mechanism of H/D exchange reactions and racemisation in aqueous solutions: configurational stability of ester and amide 
arylgycine derivatives [Kinetics and mechanism of H/D exchange reactions and racemisation in aqueous solutions: configurational stability of ester and amide arylgycine derivatives]. Cardiff: Cardiff University; 2011.

41. Narduolo S. The mechanism of racemisation of 5-substituted hydantoins in aqueous solution [The mechanism of racemisation of 5-substituted hydantoins in aqueous solution]. Cardiff: Cardiff University; 2011.

42. Ahmad HO. Kinetics and mechanism of racemisation reactions of configurationally labile stereogenic centres in drug-like molecules in aqueous solutions; thiohydantoins and related compounds [Kinetics and mechanism of racemisation reactions of configurationally labile stereogenic centres in drug-like molecules in aqueous solutions; thiohydantoins and related compounds.]. Cardiff: Cardiff University; 2015.

43. Reist M, Carrupt P-A, Testa B, Lehmann S, Hansen JJ. Kinetics and mechanisms of racemization: 5-substituted hydantoins (= imidazolidine-2,4-diones) as models of chiral drugs. Helv Chim Acta 1996;79:767-78.

44. Testa B, Carrupt P-A, Gal J. The so-called 'interconversion' of stereoisomeric drugs: An attempt at clarification. Chirality 1993;5:105-11.

45. Becke AD. Density-functional thermochemistry. III. The role of exact exchange. 1993;98:5648-52.

46. Lee C, Yang W, Parr RG. Development of the Colle-Salvetti correlation-energy formula into a functional of the electron density. 1988;37:785-89.

47. Hariharan PC, Pople JA. Influence of polarization functions on MO hydrogenation energies. 1973;28:213-22.

48. Tomasi J, Mennucci B, Cammi R. Quantum mechanical continuum solvation models. ChemRev 2005;105:2999-3094. 
49. ChEMBL. Available at: https://www.ebi.ac.uk/chembl/drugstore [Last accessed 27 November 2018]

*50. Lovering F, Bikker J, Humblet C. Escape from flatland: increasing saturation as an approach to improving clinical success. 2009;52:6752-56.

The authors demonstrate that the presence of chiral centers correlate with success as compounds transition from discovery, through clinical testing, to drugs.

51. Lambert MP, Neuhaus FC. Mechanism of d-cycloserine action: Alanine racemase from Escherichia coli W1. J Bacteriol 1972;110:978-87.

52. McClure JH. Ropivacaine. Br J Anaesth 1996;76:300-07.

53. Arvidsson T, Bruce HF, Halldin MM. Lack of metabolic racemisation of ropivacaine, determined by liquid chromatography using a Chiral AGP column. Chirality 1995;7:272-77.

54. NICE - Brinzolamide. Available at: https://bnf.nice.org.uk/drug/brinzolamide.html [Last accessed 27 November 2018]

55. Leonetti F, Capaldi C, Pisani L, Nicolotti O, Muncipinto G, Stefanachi A, et al. Solid-phase synthesis and insights into structure-activity relationships of safinamide analogues as potent and selective inhibitors of type B monoamine oxidase. JMedChem 2007;50:4909-16.

56. Development of new stereoisomeric drugs. Available at: https://www.fda.gov/drugs/guidancecomplianceregulatoryinformation/guidances/ucm $\underline{\text { 122883.htm [Last accessed } 27 \text { November 2018] }}$

57. Han J, Nelson DJ, Sorochinsky AE, Soloshonok VA. Self-Disproportionation of enantiomers via sublimation; new an truly green dimension in optical purification. Curr Org Synth 2011;8:310-17. 
58. Soloshonok VA, Roussel C, Kitagawa O, Sorochinsky AE. Selfdisproportionation of enantiomers via achiral chromatography: A warning and an extra dimension in optical purifications. Chem Soc Rev 2012;41:4180-88.

59. Sorochinsky AE, Aceña J, Soloshonok VA. Self-disproportionation of enantiomers of chiral, non-racemic fluoroorganic compounds: Role of fluorine as enabling element. Synthesis 2013;45:141-52.

*60. Sorochinsky AE, Soloshonok VA. Self-disproportionation of enantiomers of enantiomerically enriched compounds. Topics in Current Chemistry: Springer Verlag 2013:301-39.

This review discusses the often ignored potential for self-disproportionation of enantiomers in non-racemic mixtures during achiral purification techniques.

61. Han J, Wzorek A, Soloshonok VA, Klika KD. The self-disproportionation of enantiomers (SDE): The effect of scaling down, potential problems versus prospective applications, possible new occurrences, and unrealized opportunities? Electrophoresis 2019. 
Table 1. Groups identified by Testa as increasing or decreasing the stability of an adjacent stereogenic carbon. From Ref. [9]

\begin{tabular}{|c|c|c|}
\hline Decrease stability & Neutral & Increase stability \\
\hline Ester & Alkyl & Carboxylate \\
\hline Aryl ketone & & Sulfate \\
\hline \multicolumn{3}{|l|}{ Amide } \\
\hline \multicolumn{3}{|l|}{ Hydroxyl } \\
\hline \multicolumn{3}{|l|}{ Amine } \\
\hline \multicolumn{3}{|l|}{ N-linked imine } \\
\hline \multicolumn{3}{|l|}{ Halogens } \\
\hline \multicolumn{3}{|l|}{ Pseudohalogens } \\
\hline \multicolumn{3}{|l|}{ Aromatic groups } \\
\hline \multicolumn{3}{|l|}{ Benzyl groups } \\
\hline $\mathrm{CH}_{2} \mathrm{OH}$ & & \\
\hline
\end{tabular}


Table 2. Group contributions (in $\mathrm{kcal} / \mathrm{mol}$ ) for a set of substituents selected to represent more than $99 \%$ of the functional groups found adjacent to stereogenic carbon atoms in databases of compounds relevant to medicinal chemistry. Values in square brackets correspond to the deprotonated form of the substituent while those in curly brackets correspond to the protonated form.

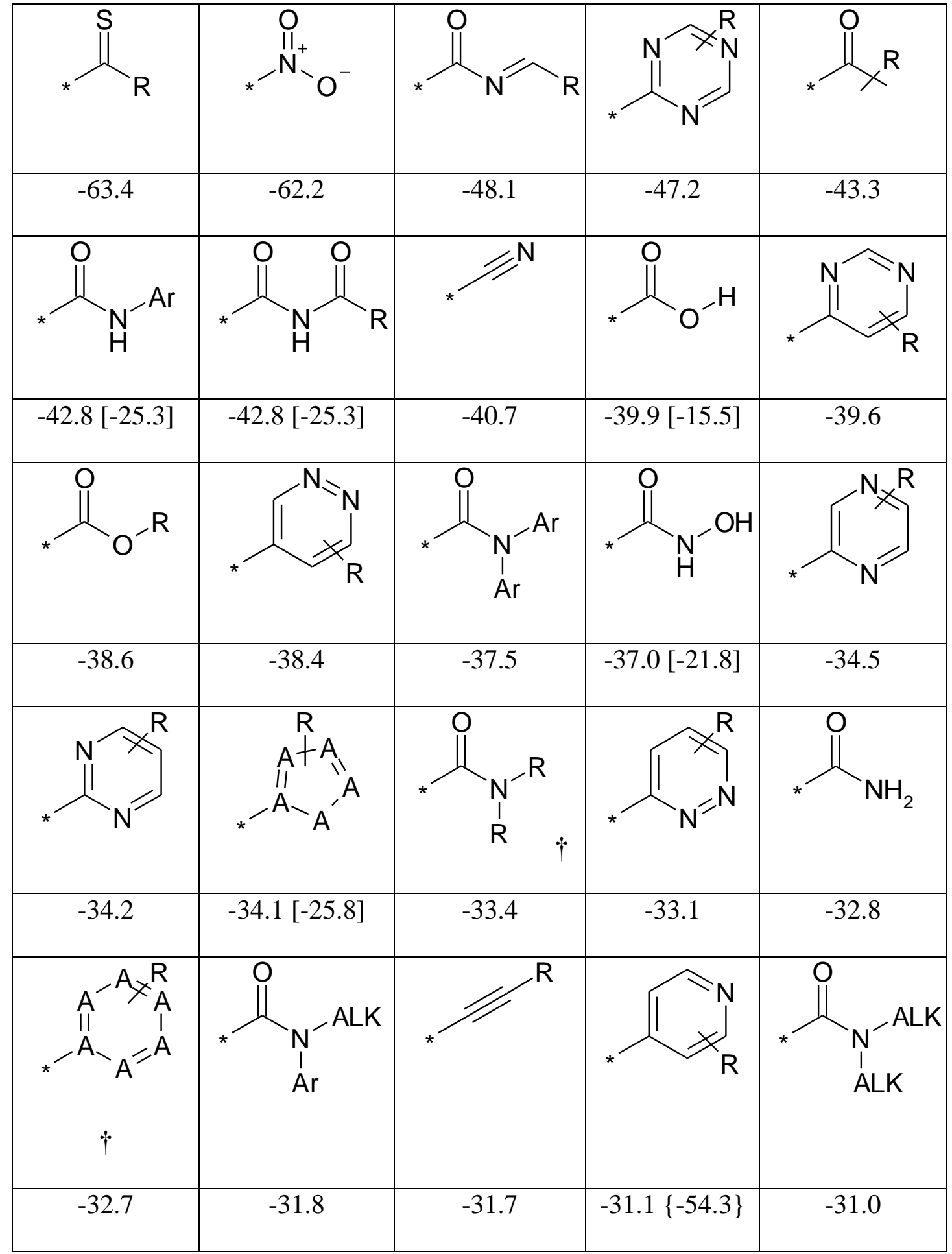




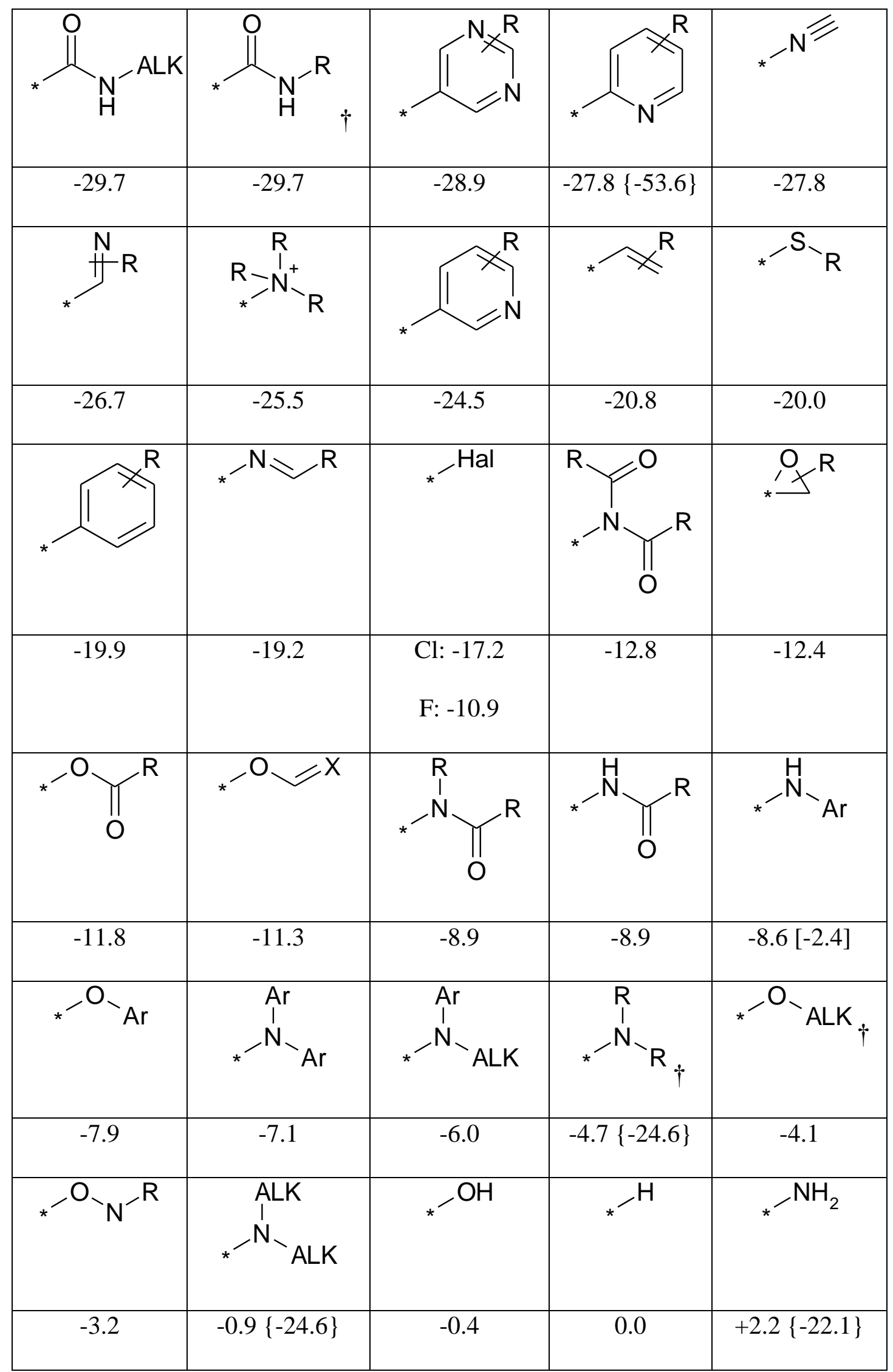




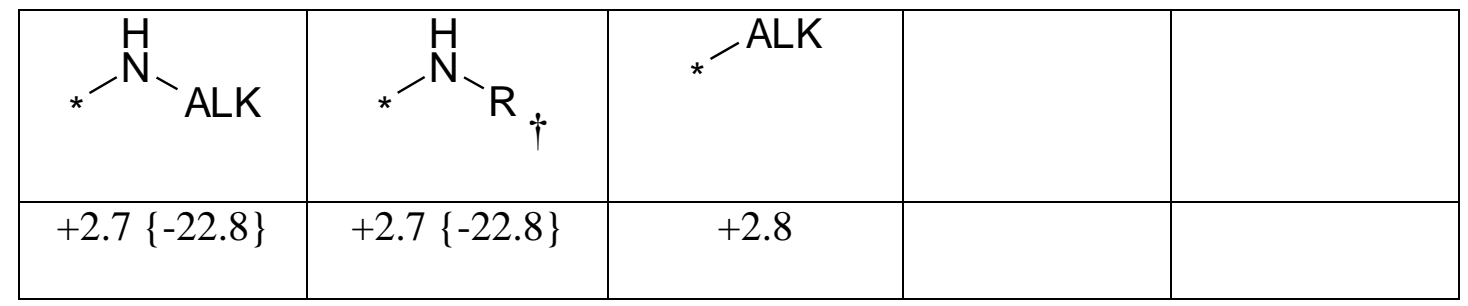

$\dagger$ If the group does not belong to one of the more specific types in the table. 


\section{CAPTIONS TO FIGURES}

Figure 1. Compounds with stereogenic centers and which are described herein.

Figure 2. A) The length of the bars corresponds to the variance attributable to inverting the chirality of a molecule and is that which is in excess of experimental variation; properties with no bar are those for which no extra variance could be discerned. Data is from Ref. [22] B) The D-value estimates the magnitude of the difference between the distribution of changes caused by inverting chirality and those for repeat testing. Bars that are solid correspond to those where a statistically significant difference was detected. Data is from Ref. [23].

Figure 3. Proposed mechanisms for racemization and related processes.

Figure 4. A) The percentage of compounds in each of four phases of clinical development that we compute to have a high likelihood of biologically relevant racemization. B) The proportion of approved drugs that belong to different compound types and the proportion belonging to different stereochemical classes for synthetic small molecules and natural product derived molecules. C) The number of compounds that we compute to have a high likelihood of biologically-relevant racemization that were approved in each of the decades shown and whether the compounds are used as racemates or single stereoisomers. 


\section{HIGHLIGHTED REFERENCES}

Of high interest:

** 2. Testa B, Vistoli G, Pedretti A, Caldwell J. Organic stereochemistry. Part 5. Helv Chim Acta 2013;96:747-98.

This review presents an in-depth and complete overview of the general principles of stereochemistry.

** 5. Islam MR, Mahdi JG, Bowen ID. Pharmacological importance of stereochemical resolution of enantiomeric drugs. Drug Saf 1997;17:149-65.

This review presents an extensive overview of the possible effects of chirality on pharmacological properties.

** 9. Testa B. Organic stereochemistry. Part 2. Helv Chim Acta 2013;96:159-88.

This review presents an in-depth and complete overview of the general principles of stereochemistry with a focus on medicinal sciences.

* 14. Ali I, Gupta VK, Aboul-Enein HY, Singh P, Sharma B. Role of racemization in optically active drugs development. Chirality 2007;19:453-63.

The authors present a detailed overview of studies of racemization of optically active drugs.

Of interest:

* 4. Caner H, Groner E, Levy L, Agranat I. Trends in the development of chiral drugs. Drug Discov Today 2004;9:105-10. 
This paper discusses trends in drug development with a focus on the effects of the 1992 FDA statement on the development of new stereoisomeric drugs.

* 12. Eriksson T, Bjorkman S, Roth B, Fyge A, Hoglund P. Stereospecific determination, chiral inversion in vitro and pharmacokinetics in humans of the enantiomers of thalidomide. Chirality 1995;7:44-52.

This paper provides rare, and therefore very valuable, kinetic data for racemization in humans.

* 50. Lovering F, Bikker J, Humblet C. Escape from flatland: increasing saturation as an approach to improving clinical success. 2009;52:6752-56.

The authors demonstrate that the presence of chiral centers correlate with success as compounds transition from discovery, through clinical testing, to drugs.

* 60. Sorochinsky AE, Soloshonok VA. Self-disproportionation of enantiomers of enantiomerically enriched compounds. Topics in Current Chemistry: Springer Verlag 2013:301-39.

This review discusses the often ignored potential for self-disproportionation of enantiomers in non-racemic mixtures during achiral purification techniques. 